\title{
eJRIEPS
}

Ejournal de la recherche sur l'intervention en éducation physique et sport

Numéro spécial 3 | 2020

Actes de la 11 ème Biennale de l'ARIS, Lille, 19-21 juin

2018

\section{Pour une éducation sensorielle à travers les activités physiques : analyse de pratique et pistes professionnelles}

Aline Paintendre, Mary Schirrer et Carole Sève

\section{CpenEdition}

Journals

Édition électronique

URL : http://journals.openedition.org/ejrieps/4382

ISSN : 2105-0821

Éditeur

ELLIADD

\section{Référence électronique}

Aline Paintendre, Mary Schirrer et Carole Sève, «Pour une éducation sensorielle à travers les activités physiques : analyse de pratique et pistes professionnelles », eJRIEPS [En ligne], Numéro spécial 3 | 2020, mis en ligne le 01 janvier 2020, consulté le 18 février 2020. URL : http://

journals.openedition.org/ejrieps/4382

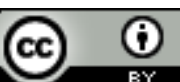

La revue eJRIEPS est mise à disposition selon les termes de la Creative Commons Attribution 4.0 International License. 


\section{Pour une éducation sensorielle à travers les activités physiques : analyse de} pratique et pistes professionnelles

Aline Paintendre ${ }^{*}$, Mary Schirrer ${ }^{* *}$ et Carole Sève ${ }^{\star * *}$

* Laboratoire Techniques et Enjeux du Corps, EA3625, Université de Paris-Descartes, France. Chercheure associée Laboratoire Interdisciplinaire des Sciences de l'Éducation et de la Communication, EA2310, Université de Strasbourg, France

** Laboratoire Interdisciplinaire des Sciences de l'Éducation et de la Communication, EA2310, Université de Lorraine, France.

*** Laboratoire Motricité, Interactions, Performance, EA 4334, Université de Nantes, France.

\section{Résumé}

Cet article présente une analyse de l'activité de lycéens en step. En référence au cadre du cours d'action (Theureau, 2006), il décrit les apprentissages sensoriels de lycéens débutants en step, et plus précisément la manière dont ils apprennent à être attentifs à leurs sensations corporelles et à les interpréter. L'enjeu est de mieux comprendre les processus de construction de "savoir-faire perceptifs " chez les pratiquants, en nous référant à leur vécu. En effet, la construction de compétences perceptives et sensibles apparaît fondamentale chez le pratiquant pour réguler les actions motrices, apprendre à gérer l'effort, mais aussi dans une logique de développement du bien-être et de la santé. Aussi, il s'agit d'explorer le développement de savoir-faire perceptifs, afin de proposer des stratégies d'intervention et des postures d'accompagnement singulières pour des acteurs de l'éducation par les APSA.

Mots clés : éducation et apprentissages sensoriels, savoir-faire perceptif, step, Éducation Physique et Sportive, compétence perceptive et sensible.

\section{Summary}

This article presents an analysis of students' activity in step. Referring to the theoretical and methodological framework "course of action" (Theureau, 2006), it describes their sensory learnings in step. It is focused on the way students learn to pay attention to their body's sensations, and how they interpret them. The aim of this study is centered on the 


\section{eJRIEPS spécial 3 (janvier 2020)}

construction processes of "perceptive know how" by practitioners, by referring to their experiences. Indeed, building perceptual and sensitive competences seems fundamental to the practitioner, in order to regulate his actions, to better regulate his effort, and for enhancing health and well-being. Finally, this research explores how perceptive know-hows can be developed. From this point onwards, some interventions strategies and singular accompaniment postures can be proposed for actors of education through physical practices.

Key words: Sensory Education and Learning, Perceptual know-how, step, Physical Education, Perceptual and Sensitive Competence.

\section{Introduction}

Depuis quelques années des pratiques physiques orientées vers la conscience de soi et inspirées de pratiques orientales, telles que la méditation, le qigong, le yoga et les arts martiaux, se développent. Des pratiquants valorisent également l'écoute de leur intériorité en choisissant des sports tels que l'apnée (Schirrer, 2015). L'Éducation Physique et Sportive (EPS) n'est pas restée étrangère à ce mouvement de retour sur soi dans les pratiques physiques. L'introduction de la compétence propre "Réaliser et orienter son activité physique en vue du développement et de l'entretien de soi » dans le programme EPS de lycée de 2010 (BO spécial $n^{\circ} 4$ du 29 avril 2010) vise, entre autres, à centrer l'attention des élèves sur leurs ressentis, afin qu'ils apprennent à réguler leur effort sur la base de leur prise en compte. Dans le même mouvement, le yoga vient d'être introduit dans la liste nationale des activités physiques sportives et artistiques, dans le programme EPS 2019 du lycée (BO spécial n¹ du 22 janvier 2019).

La sensorialité et l'écoute de soi nous semblent constituer des enjeux d'importance pour un développement harmonieux de la personne, dans un monde où le corps tend de plus en plus à être appréhendé et piloté par des objets connectés (Verchère, 2016). Le développement, par la pratique d'activités physiques, de savoir-faire perceptifs orientés vers l'intériorité corporelle semble directement connecté à un enjeu sociétal majeur de bien-être et de santé. Dans un contexte d'objectivation des corps, d'hyper-responsabilisation des sujets vis-à-vis de corps mesurés et contrôlés, il s'agit d'aider les pratiquants à faire un " bon usage de soi » en devenant «agent de soi » (Andrieu, 2012). II s'agit d'apprendre à 


\section{eJRIEPS spécial 3 (janvier 2020)}

focaliser l'attention sur les données sensorielles qui accompagnent toute expérience corporelle, afin de mieux connaître et respecter les possibilités de son corps. Par une nouvelle intelligibilité corporelle, ces savoir-faire peuvent renforcer le plaisir lié à la sensorialité et aider le pratiquant à se réapproprier son corps. Ils seraient ainsi au centre d'une éducation sensorielle, susceptible d'enrichir les pratiques d'intervention en EPS et en sport.

Si les travaux de Schirrer (2015) analysent les stratégies d'intervention d'entraineurs afin de favoriser la constitution de savoir-faire perceptifs chez des apnéistes, notamment par la co-construction d'une nouvelle grille de lecture de son expérience corporelle et sensible, la prise en compte de la sensorialité du pratiquant dans les processus d'apprentissage (dans le monde scolaire et sportif) est cependant peu explorée et formalisée que ce soit dans la littérature scientifique ou professionnelle. Comment les pratiquants développent-ils leur capacité, au cours de la pratique, à être attentifs à leurs sensations corporelles et à les interpréter ? Comment les intervenants peuvent-ils aider les pratiquants dans le développement de leur sensorialité ?

L'objectif de cet article est de présenter les résultats principaux d'une étude en step s'intéressant à des « apprentissages sensoriels » chez des pratiquants novices afin d'interroger les apports de la notion de savoir-faire perceptifs déjà questionnée dans la pratique de l'apnée (Schirrer, 2015) et de proposer des pistes d'intervention pour une éducation sensorielle dans le monde scolaire voire sportif.

\section{Synthèse de littérature, ancrages théoriques et question de recherche}

\subsection{Sensation et perception}

La sensation résulte de la stimulation, externe ou interne, des récepteurs sensoriels. Elle peut donc rester à l'état diffus. Le rapport à l'environnement par la perception du sujet s'établit à partir d'une multimodalité sensorielle où différents capteurs convergent sur une même temporalité (Berthoz, 2013). L'homme dispose de deux sources d'informations pour s'orienter dans le monde : extéroceptives (les cinq sens : vision, audition, olfaction, goût et toucher) qui parviennent de l'environnement dans lequel il évolue ; et intéroceptives qui parviennent de l'intérieur de lui-même, par des récepteurs sensoriels situés dans les tissus et tout un ensemble de cellules constituant la proprioception (Gaillard, 2011), et par l'ensemble des informations viscérales. Les études en neurosciences (Craig, 2003, 2004 ; Pollatos, Schandry, Auer, \& Kaufmann, 2007 ; Farb, Segal, \& Anderson, 2012) nous apprennent que les mécanismes endogènes de contrôle homéostatique modulent l'activité 


\section{eJRIEPS spécial 3 (janvier 2020)}

et l'état du corps, les sensations perçues et les sentiments corporels (Gaser \& Schlaug, 2003). Lors de l'activité physique, ces informations renseignent par exemple sur l'état de chaleur du corps, l'état des organes tels que le cœur ou le système respiratoire.

La perception renvoie à ce qui est perçu de la sensation, elle est une "exploration active " (Berthoz, 2013, p. 179). Elle résulte de l'interprétation et du jugement que nous faisons à partir de la sensation. Le terme « ressenti » renvoie plus communément à la capacité d'une personne à se rendre attentive à ses sensations internes. Ainsi, la qualité perceptive dépend de l'attention portée aux sensations corporelles : une disposition à une « présence attentive " à ses sensations ou encore un processus d'“ awareness " (Fourure, 2004). En effet, si les perceptions sont orientées par les potentialités des récepteurs, les significations diverses acquises par chaque individu au cours de son existence interviennent aussi (Wathelet, 2013). En d'autres termes, ce que nous percevons est l'objet d'une visée de la conscience : nous ne percevons que ce que nous sommes disposés à percevoir. Bouveresse (1995) distingue sensation et perception : des sensations différentes peuvent donner lieu à des perceptions identiques, tout comme des sensations identiques peuvent aboutir à des perceptions différentes. Qualité intrinsèque de tout tissu vivant, la sensibilité est aussi cette capacité ou disposition de l'homme à être affecté par ses sens, des variations de ses états internes ou de certains aspects de l'environnement (Récopé, 2001 ; Gaillard, 2011). L'expérience sensible est causée par l'environnement, et elle cause à son tour le jugement, la formation de croyances perceptuelles (Bouveresse, 1995).

\subsection{Phénoménologie : interroger le corps par le prisme de la sensibilité}

En nous référant à Merleau-Ponty (1945), nous considérons le corps phénoménal comme percevant et sensible en rapport direct et immédiat avec son environnement (Varela, 1989). En d'autres termes, ce corps phénoménal représente le corps perceptif, qui émerge à la conscience et est doté d'une sensibilité singulière. La sensibilité représente la qualité du corps sentant. Elle est une forme d'interface entre l'acteur et son monde. Gaillard (2011) relève qu'elle est un « moyen d'information », un « fond sensoriel » et exige un cheminement de la part de l'acteur pour devenir attentif aux possibilités de son corps. En effet la sensibilité peut s'affiner par une plus grande ouverture attentionnelle à ses sensations (Bois \& Austry, 2007) et en devenant un «visiteur assidu » de son corps (Da Silva, 2013). Cette hyperacuité est le fruit d'un apprentissage (Bois \& Austry, 2007) où les « qualités sensibles », contribuent peu à peu à qualifier et cartographier les sensations corporelles. Des " connaissances sensibles » (Barberousse, 1999) ou « connaissances par corps " (Vanpoulle, 2013) se 


\section{eJRIEPS spécial 3 (janvier 2020)}

construisent et s'actualisent dans l'action par les retours répétés de certaines sensations. Elles favorisent un auto-ajustement permanent de la part de l'acteur pour constituer et affiner un répertoire sensible. En effet, " ces connaissances incorporées, (...) sont typiquement le fruit d'une expérience corporelle fondée sur le ressenti. (...). Dans le processus d'apprentissage en EPS, les connaissances nouvelles émergent de mises en relation entre des connaissances acquises et un vécu corporel riche de sensations. " (Huet \& Gal-Petitfaux, 2011, p. 64-65).

\subsection{La notion de savoir-faire perceptif}

\subsubsection{Définition}

De nombreux champs disciplinaires investiguent les notions de perception et sensations corporelles et l'intérêt d'un ancrage épistémologique pluridisciplinaire (Collinet, Terral, \& Trabal, 2016) repose sur la prise en compte du corps dans une acception holistique (Vanpoulle, 2013 ; Andrieu, 2012, 2017). Nous proposons et interrogeons la notion de savoir-faire perceptif (Wathelet, 2013 ; Schirrer \& Paintendre, 2017) qui nous semble pertinente pour questionner la place qu'occupent les sensations dans les capacités corporelles (Paintendre, 2017). Par savoir-faire perceptif, nous entendons une capacité à écouter et interpréter les sensations qui émanent de son corps, dans un dessein d'adaptation à l'environnement et/ou de meilleure gestion de son capital corporel (Schirrer \& Paintendre, 2017 ; Paintendre, Schirrer, \& Andrieu, 2019). Envisagée en tant que processus, la sensibilité, disposition de l'homme à être affecté par ses sens, pourrait se modeler, s'actualiser, s'ajuster sans cesse (Wathelet, 2013). Différentes études, en sociologie et anthropologie sensorielle notamment, offrent des éléments de compréhension sur la manière dont les individus apprennent de nouvelles configurations sensorielles, de nouveaux usages et langages des sens (Becker, 2012 ; Schirrer, 2015, 2018). Ces travaux s'intéressent à la perception comme acte, afin de mettre en avant des processus de socialisation de l'attention, d'éducation de la sensibilité.

\subsubsection{Savoir-faire perceptifs et stratégies d'intervention}

Schirrer (2015), par une démarche ethnographique, révèle les stratégies d'intervention de l'entraineur pour favoriser la constitution de savoir-faire perceptifs chez l'apnéiste. Elle montre aussi que les connaissances physiologiques acquises par le pratiquant débutant en apnée lui permettent d'intégrer ses différentes sensations dans un réseau de significations (différentes phases d'une apnée). Celui-ci comprend intellectuellement la phase qu'il lui faut 


\section{eJRIEPS spécial 3 (janvier 2020)}

maîtriser en compétition mais les mises en œuvre sont encore difficiles. Si l'explication physiologique apportée par l'entraineur et les pairs semble informer l'entrée sensorielle, c'est bien la pratique et la réflexivité sur cette pratique qui permettent au pratiquant de construire et d'affiner des repères sensoriels.

Inspirée par une démarche d'éducation sensorielle proche de la Programmation NeuroLinguistique (PNL), l'une des stratégies d'intervention repose sur une aide à l'identification des instants clés dans sa pratique en apnée. II s'agit de " favoriser l'hyper-écoute de son corps et de son mental par l'apnéiste »(Schirrer, 2018, p. 41). La médiation langagière apparaît essentielle afin d'aider les pratiquants à analyser ce qu'ils ont vécu en éduquant l'attention des pratiquants sur des traits pertinents de leurs sensations. Par la pratique de l'apnée, les pratiquants vivent des modelages sensoriels. Ces modelages visent à les rendre plus attentifs à certaines sensations, à les percevoir, à les interpréter ou juger, c'està-dire les faire entrer dans un univers de significations partagé. Ils construisent ainsi des savoir-faire perceptifs et sensibles, c'est-à-dire une capacité à identifier des sensations corporelles et intéroceptives, pour agir et prendre des décisions. Focalisation de l'attention, « extrémisation » des sensations à l'aide de situations contrastées, partage des ressentis, médiation langagière, utilisation de métaphores pour dire le vécu corporel, apport de connaissances théoriques et proposition puis affinement d'une échelle de ressentis sont autant de démarches proposées par l'entraineur en apnée (Schirrer, 2018), destinées à faciliter cette éducation sensorielle pour faire entrer les pratiquants dans une posture d'écoute attentive de leur corps.

\subsection{Step scolaire : une pratique physique au service d'une écoute de ses} sensations corporelles

Du fait de ses caractéristiques, le step scolaire est une situation d'étude privilégiée pour appréhender la construction de savoir-faire perceptifs. Le step est une pratique physique de montée et de descente de marche sur un support fixe (stepper). Elle se pratique en musique pour déterminer tempo et exécution des mouvements (Paintendre, 2015). La pratique du step en EPS vise un engagement des élèves dans un effort physique pour obtenir des effets recherchés sur soi selon un projet personnel et en relation avec un thème d'entraînement choisi. Ces thèmes correspondent à des intensités d'effort variables par l'ajustement de paramètres énergétiques et biomécaniques. La pratique du step exige une attention à ses sensations corporelles et a pour enjeu une amélioration de la connaissance de soi des élèves, notamment par la construction « de liens explicites » entre des informations internes 


\section{eJRIEPS spécial 3 (janvier 2020)}

et objectives (Boulnois \& Kogut, 2014). Pour ce faire, les enseignants recourent à deux outils principaux: le carnet d'entraînement et le cardiofréquencemètre. Le carnet d'entraînement de l'élève comporte des échelles de ressentis, souvent proposées par l'enseignant (Coquart, Lensel, \& Garcin, 2009 ; Garcin, 2002), afin d'aider les élèves à planifier, réguler et analyser leur projet de pratique personnelle. Le cardiofréquencemètre permet de réaliser des relevés en direct de la fréquence cardiaque qui est un indicateur de l'intensité de son engagement physique.

\subsection{Problématique et question de recherche}

Nous avons analysé l'activité de lycéens débutants dans la pratique du step. Dans un cadre scolaire, au cours d'une séquence d'apprentissage en step, nous avons exploré leurs apprentissages sensoriels. Sur la base de quelles informations les élèves régulent-ils leur niveau d'engagement physique en step ? Leurs perceptions sensorielles sont-elles des informations fiables ? Comment les élèves parviennent-ils à articuler les données du cardiofréquencemètre et leurs ressentis ? L'enjeu est de mieux comprendre les processus de construction de savoir-faire perceptifs de débutants en step, en nous référant à leur vécu, pour proposer des pistes d'accompagnement des pratiquants vers une meilleure écoute de leur corps.

\section{Méthodologie de recherche : cours d'expérience}

Notre méthodologie d'étude s'appuie sur le cadre théorique et méthodologique du cours d'action (Theureau, 2006, 2010) qui a été exploité dans diverses études sur l'activité des élèves (e.g., Saury et al., 2013). Nous nous sommes appuyés sur l'objet théorique du « cours d'expérience » qui exprime l'activité telle qu'elle est vécue par l'acteur « selon un grain très fin, et en particulier (...) ses détails sensoriels et émotionnels " (Theureau, 2010, p. 299). II est constitué de l'enchaînement d'unités d'expérience qui permettent de rendre compte de l'évolution des préoccupations de l'acteur, de ses attentes, des connaissances mobilisées et construites, des éléments pris en compte pour agir, au cours de périodes d'activité. La comparaison des unités d'expérience à différents moments permet ainsi de caractériser des phénomènes d'apprentissage. Le cours d'expérience est reconstruit sur la base de différentes données : des données objectives (traces de l'activité de l'acteur tels que des enregistrements audio-visuels, des prises de notes, etc.) et subjectives (verbalisations recueillies lors d'entretiens d'autoconfrontation au cours desquels l'acteur, 


\section{eJRIEPS spécial 3 (janvier 2020)}

confronté à l'enregistrement audio-visuel de son activité est invité à la commenter sans la rationnaliser).

Nous avons réalisé notre étude au sein de deux classes de première générale, lors d'une séquence d'enseignement de dix leçons de step, avec douze lycéens volontaires et débutants. Nous avons observé le déroulement des leçons et l'activité des élèves lors de l'ensemble de la séquence, et tenu un cahier d'observation. Nous avons utilisé une caméra Go Pro (C) et une caméra positionnée pour une visualisation de la classe en plan large, afin de recueillir diverses traces audiovisuelles de l'activité des élèves. Des entretiens d'autoconfrontation (Theureau, 2010) d'une durée moyenne de 52 minutes ont été réalisés lors de la leçon 1 (début de séquence) et lors de la leçon 7 (fin de séquence) afin d'étudier le processus d'apprentissage des élèves au cours de la séquence (pour plus de détails, voir Paintendre, Schirrer, \& Andrieu, 2019). Les carnets d'entraînement construits par l'enseignante et renseignés par les élèves au cours de chaque leçon (ressentis, paramètres de l'entraînement en step) ont été mobilisés lors des entretiens par les lycéens pour les aider à exprimer leur vécu.

De plus, une mesure continue de l'activité cardiaque des élèves a été réalisée sur l'ensemble des leçons grâce à l'équipement d'un cardiofréquencemètre de type Polar (ceinture thoracique). Au cours de chaque leçon, les élèves accèdent aux données du cardiofréquencemètre en direct et sont invités dans le carnet d'entrainement à exprimer leurs ressentis à l'aide d'échelles construites par l'enseignante. Pendant les séquences de travail, l'intensité d'effort du pratiquant doit être supérieure ou égale à un pourcentage de la Fréquence Cardiaque d'Entrainement (FCE) selon le thème d'entrainement choisi ( $B O{ }^{\circ} 25$ du 21 juin 2018). La formule de Karvonen définit la FCE : FC Repos + [(FC Max - FC Repos) $x \%$ d'intensité de travail définie selon le thème d'entraînement]. Le traçage de la fréquence cardiaque est effectué seconde après seconde.

L'ensemble de ces données (entretiens, enregistrements audio-visuels, carnets d'entraînement, observations in situ, relevés de fréquence cardiaque) a permis de reconstruire, pour les leçons 1 et 7, le cours d'expérience (Theureau, 2006) des élèves. Nous nous sommes plus particulièrement intéressés aux préoccupations des élèves, aux éléments pris en compte pour agir, et aux connaissances mobilisées et construites, afin de caractériser l'évolution, au cours de la séquence d'enseignement, de la capacité des élèves à prendre et interpréter des informations (sensorielles ou objectives) pour juger et réguler leur niveau d'engagement physique. La mise en correspondance d'extraits des cours 


\section{eJRIEPS spécial 3 (janvier 2020)}

d'expérience avec les relevés du cardiofréquencemètre a permis d'évaluer la fiabilité des jugements des élèves concernant l'intensité de leur effort physique.

\section{Résultats}

\subsection{Une préoccupation relative à l'écoute de son corps}

L'analyse de l'activité des élèves révèle des préoccupations plurielles relatives à une recherche d'efficacité de l'action, à l'optimisation du rapport capacités corporelles-intensité d'effort et à l'écoute de son corps. Dans le cadre de cet article, nous détaillons cette dernière préoccupation qui se traduit par une centration sur soi, notamment par une concentration sur son effort « je suis attentive à ce que je fais, sur l'effort que je fais », ou par une centration sur des sensations corporelles telles que la respiration "j'essaie de pas expirer, enfin vraiment vite comme si on courait », "j'étais de nouveau concentrée sur comment était ma respiration » ou l'état de chaleur « ça me donnait encore plus chaud de faire ça, je me focalise là-dessus ».

Quand les élèves sont préoccupés par cette écoute du corps, ils mobilisent et construisent des connaissances qui reposent sur la perception et l'interprétation de certaines sensations corporelles : des sensations musculaires au niveau des jambes (cuisses, mollets) « mes jambes sont lourdes, ça devient beaucoup d'effort de juste lever la jambe », « je sens que ça me tire », les battements du cœur (carotide, poitrine) « on sent les battements, ça vient de l'intérieur », des sensations de chaleur (tête, corps) « il fait chaud au visage », "ça me brûle, je le sens à l'intérieur, et c'est très désagréable », ou encore des sensations respiratoires « je respire un peu plus difficilement, il faut que j'inspire plus fort, que j'expire plus fort, pour la même quantité, enfin je sais pas trop comment expliquer ça ». Cette préoccupation d'écouter son corps coïncide avec la constitution d'un projet personnel de pratique en step, dans la mesure où les élèves cherchent à lier des informations objectives (fréquence cardiaque, posture lors de la réalisation du pas) et des informations internes (ressentis). En effet, différents paramètres d'entrainement leur sont proposés lors des leçons pour les conduire à comparer les effets de la pratique sur leur corps et construire différentes connaissances sur soi lors de la séquence d'apprentissage.

L'analyse révèle que la capacité à prendre en compte ses sensations corporelles pour évaluer et réguler l'intensité de son effort physique diverge selon les élèves. Pour juger de leur état corporel, les élèves prennent en compte des éléments divers et construisent des connaissances plus ou moins fiables. L'acuité perceptive des élèves ou encore cette capacité à écouter son corps s'inscrit dans un processus d'apprentissage différencié, qui se 


\section{eJRIEPS spécial 3 (janvier 2020)}

manifeste particulièrement lors de la comparaison des données du cardiofréquencemètre et du cours d'expérience des pratiquants.

\section{2. Études de cas}

Afin d'illustrer les différences entre les élèves, nous présentons deux études de cas extraites de la leçon 7 de la séquence d'enseignement. Elles concernent Emmi et Maeva, toutes deux novices dans l'activité step et respectivement engagées dans un projet d'entraînement de type puissance et capacité aérobie.

\subsubsection{Emmi : des sensations globales et diffuses}

Lors de la leçon 7, Emmi travaille le thème d'entrainement "puissance aérobie " qui nécessite un engagement physique relativement intense. Elle doit réaliser deux séries de 12 répétitions de 1 minute de travail, à une intensité supérieure ou égale à $80 \%$ de sa FCE, soit une fréquence cardiaque minimale de $173 \mathrm{bpm}$. Lors de la onzième répétition de sa série 1, Emmi est totalement investie dans son effort et elle cherche à mobiliser la totalité de son corps "Je suis vraiment en train de bouger toutes les parties de mon corps pour faire le plus de mouvements possibles et augmenter au max la FC... j'essaie de faire de grands mouvements ». Elle souhaite réaliser les « mouvements les plus grands et amples possible », afin d'augmenter sa fréquence cardiaque et atteindre l'objectif fixé : «j'essaie de faire mon maximum pour atteindre le minimum que l'on doit dépasser ».

Pour juger de l'intensité de son effort, elle s'appuie sur une impression générale " je sens que je suis vraiment en mouvement " mais aussi sur certains indicateurs tels que des sensations corporelles liées à un état de chaleur " mais déjà là, j'avais très chaud ", "j'étais beaucoup en sueur » et des informations kinesthésiques relatives à l'amplitude de ses mouvements : " ma jambe aussi, je dois le sentir au niveau de la cuisse. (...) je sens que bouge un peu le haut du corps aussi ». Elle fait également référence à des sensations antérieures pour apprécier son niveau d'engagement « je sens que j'ai fait mieux qu'avant ». Bien qu'elle ait une impression « d'épuisement » et de «bouger beaucoup » qui repose sur des connaissances construites à partir de cet ensemble de sensations corporelles perçues, Emmi n'est pas certaine d'être au maximum de la mobilisation effective de ses potentialités maximales : « je ne sens pas forcément que c'est le maximum ». Lors de cette répétition, Emmi, compte-tenu de la nécessité de la rapidité d'enchaînement des mouvements (notamment les bras) ne consulte pas son cardiofréquencemètre. 


\section{eJRIEPS spécial 3 (janvier 2020)}

Tableau I : Unité d'expérience n4, Emmi, leçon 7, répétition 11, période de 20 " à $30^{\prime \prime}$

\begin{tabular}{|c|}
\hline $\begin{array}{l}\text { Unité d'expérience d'Emmi } \\
\text { Répétition } 11 / 12 \text {, série } 1 \text {, de } 20 \text { " à } 30 \text { " d'effort }\end{array}$ \\
\hline $\begin{array}{l}\text { Préoccupations } \\
\text { - } \quad \text { S'engager physiquement en mobilisant la totalité de son corps et en faisant de grands mouvements } \\
\text { - } \text { Apprécier son niveau d'engagement physique par rapport à la fréquence cardiaque cible } \\
\text { Eléments pris en compte pour agir } \\
\text { - } \quad \text { Sensation de mouvement } \\
\text { - } \quad \text { Amplitude des mouvements réalisés } \\
\text { - } \quad \text { Sensation de chaleur } \\
\text { - } \quad \text { Sueur } \\
\text { Connaissances mobilisées et construites } \\
\text { - } \quad \text { Référence à des sensations antérieures (mobilisée) } \\
\text { - Faire des grands mouvements augmente l'intensité de l'effort (construite) }\end{array}$ \\
\hline $\begin{array}{l}\text { Description extrinsèque de l'activité d'Emmi } \\
\text { (Par le chercheur sur la base de l'enregistrement audio-visuel) }\end{array}$ \\
\hline $\begin{array}{l}\text { A 20" effort sur la séquence 11, Emmi débute son bloc } 1 \text {. Emmi est sur le tempo, les bras sont engagés et } \\
\text { accompagnent le mouvement. Elle réalise à } 20 " \text { le stomp sans propulsion avec un engagement des bras, un } \\
\text { buste assez droit et un trajet en orientation au dos du step; puis le kick est réalisé avec impulsion et } \\
\text { accompagnement des bras, regard fixé devant (on voit la tête droite); tap up sans impulsion puis mambo et } \\
\text { regard coté camarade de droite et poursuite du mouvement puis pivot, toujours engagement des bras; on voit } \\
\text { Emmi compter sur la fin du bloc, lors du retour du pivot notamment et le début du bloc } 2 \text { côté gauche, jambe } \\
\text { droite; l'ensemble du bloc est assez ample avec appui sur le step aux extrémités de celui-ci; }\end{array}$ \\
\hline
\end{tabular}

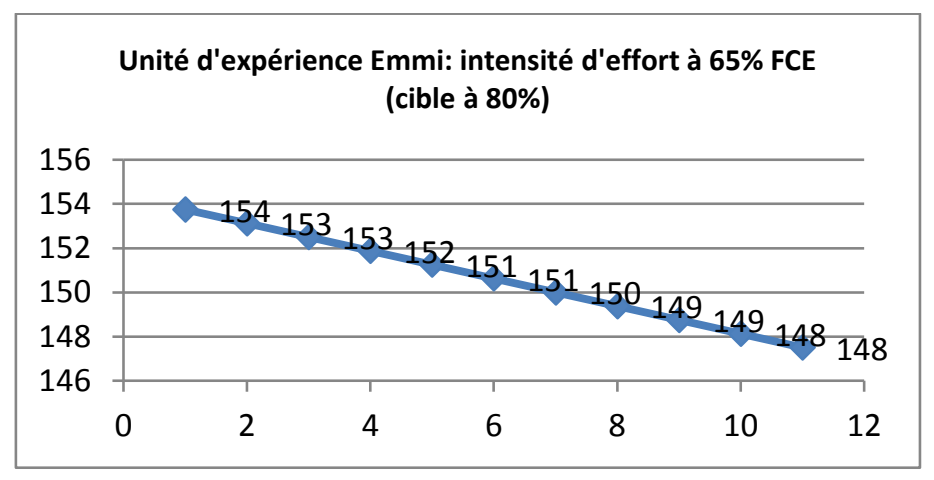

Figure 1: Relevé de fréquence cardiaque d'Emmi lors de la répétition 11 de 1 minute, période de 20 " à 31 "

Emmi a des difficultés à estimer précisément l'atteinte de son objectif en termes d'intensité et de fréquence cardiaque cible. Bien que, sur la base des sensations corporelles 


\section{eJRIEPS spécial 3 (janvier 2020)}

ressenties, elle estime son effort physique conséquent, le relevé de fréquence cardiaque indique une sollicitation énergétique à hauteur de $65 \%$ de sa FCE (au lieu de $80 \%$ de la FCE recherchée) (Figure 1). Par rapport à la valeur moyenne de son intensité physique lors de cette leçon (134 bpm), Emmi réalise un engagement supérieur. Toutefois, son effort n'est pas encore maximal puisqu'au cours de cette leçon, elle parvient à atteindre une FC de 191 bpm, soit plus de $90 \%$ de sa FCE lors de $10^{\text {ème }}$ répétition de 1' de la seconde série.

Aussi, il semble qu'Emmi ne parvienne pas à juger de manière fiable son niveau d'engagement physique réel sur la seule base des connaissances construites reposant sur ses sensations corporelles. Elle montre une capacité à percevoir des sensations corporelles encore relativement diffuses, liées notamment à un état de chaleur du corps, mais ne parvient pas par exemple à situer ces sensations dans des zones corporelles. Elle est encore en phase de découverte de ses possibilités corporelles et en quête d'informations sensorielles fiables pour moduler son intensité d'engagement. Pour ce faire, Emmi doit encore apprendre à établir des liens explicites entre les données du cardiofréquencemètre (qui la renseignent sur la réalité de l'intensité de son effort physique) et ses propres ressentis, et par l'utilisation des échelles de ressentis du carnet d'entrainement afin de l'aider à discriminer et hiérarchises les sensations perçues.

\subsubsection{Maeva : une discrimination dans les sensations}

Lors de la leçon 7, Maeva cherche à atteindre l'objectif déterminé par le thème d'entrainement « travail en capacité aérobie » qui nécessite des efforts relativement longs à une intensité modérée à élevée. Maeva doit réaliser 3 répétitions de 8 minutes à un effort d'intensité compris entre $70 \%$ et $80 \%$ de la FCE, soit une fréquence cardiaque ciblée entre 159 et 173 bpm. Lors de la 2ème répétition, elle regarde régulièrement son chronomètre afin de connaître le temps de travail restant « je voulais regarder mon temps... parce que je commence à être fatiguée ». Néanmoins, elle ne relève pas les données de son cardiofréquencemètre. Elle explique qu'en même temps, elle cherche à écouter ce qui se passe dans son corps. Ayant déjà réalisé un peu plus de la moitié de son temps d'effort (4'12"), Maeva « attend » la fin de sa répétition de 8 minutes.

Elle ressent un ensemble de diverses informations sensorielles (respiratoires, musculaires) qu'elle met en relation et hiérarchise, et qui la renseignent quant à l'intensité de son effort physique :

« II y a la respiration surtout et après les jambes...les jambes sont lourdes... à un moment ça ne suit plus parce que là, j'avais envie de m'arrêter mais je vais 


\section{eJRIEPS spécial 3 (janvier 2020)}

continuer et c'est que je vais devoir prendre sur moi et, et continuer même si ça commence à être lourd et difficile au niveau de la respiration ».

Elle semble percevoir et juger très exactement de son niveau d'intensité physique. Elle explique se passer de la lecture de son cardiofréquencemètre pour vérifier l'atteinte de la FCE (entre $70 \%$ et $80 \%$ ) du fait de la confiance qu'elle accorde à ses connaissances construites sur ses perceptions sensorielles.

Elle rapporte : «c'est vrai que c'est assez secondaire (...) oui moi je ne me focalise pas trop là-dessus parce que de toute façon les sensations... enfin, l'indicateur de la FC c'est quand même moins fiable que mes impressions à moi (...) en plus j'y étais à chaque fois dedans donc c'est construit ». En montrant son cœur, " oui oui oui c'est là que ça se passe (...) on sent les battements quand ça va vraiment fort, quand t'es vraiment au bout, moi je le sens parce que ça vient de l'intérieur »

Tableau II : Unité d'expérience $n^{\circ} 6$, Maeva, leçon 7, répétition 2 sur 3 de 8 minutes d'effort

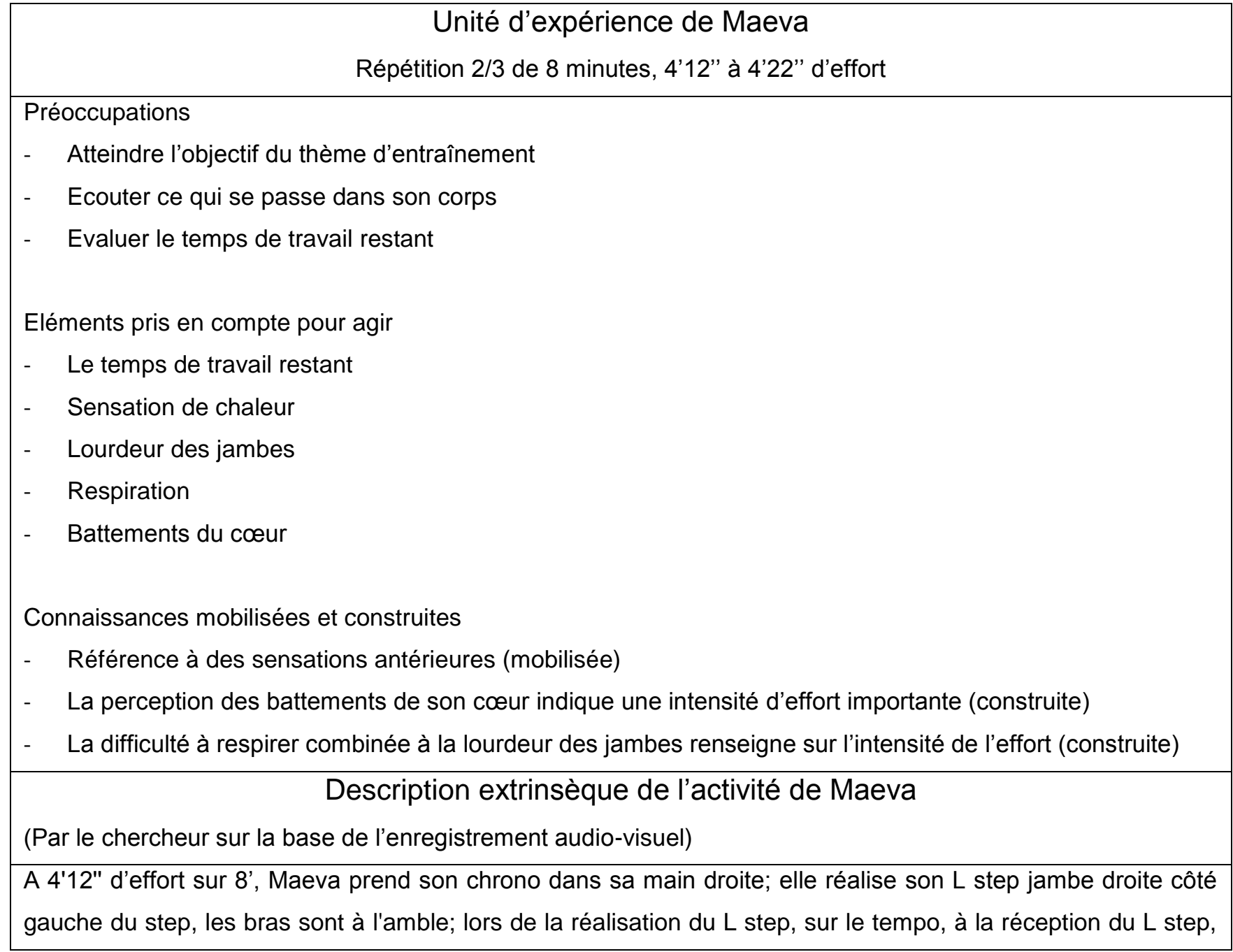




\section{eJRIEPS spécial 3 (janvier 2020)}

Maeva est légèrement décalée vers la gauche du step comme pour pré-orienter pour le pas suivant; à 4'16", elle regarde encore son chrono et réalise un stomp avec un 1er appui bien à l'extrémité puis un second qui engage l'orientation un peu trop au centre; les bras sont ballants, le buste assez droit; la pose du pied en entier sur le step; lors du 3ème appui sur le step, la pose est centrale; le kick qui suit échoue et son entame est encore au centre

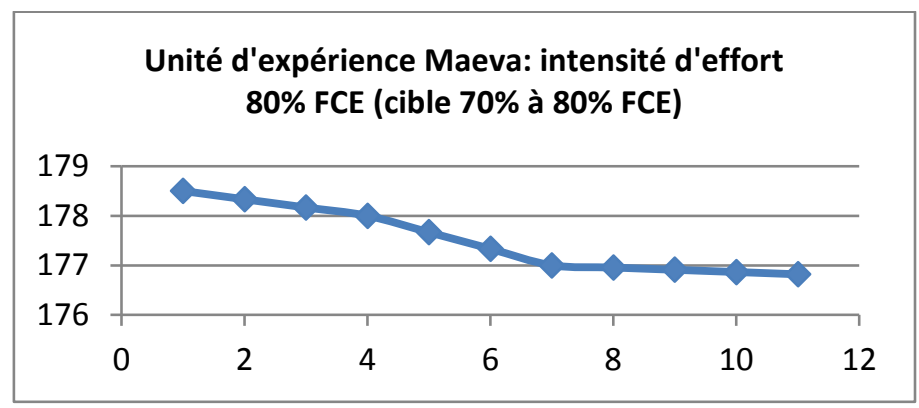

Figure 2 : Relevé de fréquence cardiaque de Maeva lors de la répétition 2/3 de 8 minutes, période de 4'12"' à 4'23"

Le relevé du cardiofréquencemètre confirme que Maeva est engagée à une intensité d'effort relativement importante avec une FC stabilisée entre 179 et 177 bpm au cours de cette unité d'expérience. Cela représente quasiment la FC maximale atteinte lors de la leçon 7 (180 bpm). Par conséquent, à ce stade de la répétition, le jugement de Maeva sur son niveau d'intensité d'effort correspond bien à la réalité. Maeva fait preuve d'une grande qualité d'écoute corporelle, et a confiance en son jugement sur la base des informations internes ressenties. La valeur minimale de la FCE cible (157 bpm) est atteinte précocement pendant la répétition de 8 minutes, augmentant sans doute l'intensité des sensations corporelles, et permettant de renforcer leur perception, notamment les battements du cœur. Pour apprécier son niveau d'engagement physique, Maeva se fie davantage à ses sensations internes, perçues pendant l'effort qu'aux données objectivées par le cardiofréquencemètre, auxquelles elle ne se réfère pas pendant l'effort malgré le regard porté à son chronomètre. Cette sensibilité relativement fine à partir d'informations sensorielles justement évaluées par Maeva, montre la place d'une lecture incarnée pendant l'effort pour réguler et ajuster sa pratique. In fine, elle valide ses connaissances sur soi constituées à partir de ses sensations corporelles. Bien qu'elle identifie au niveau de ses jambes une capacité à poursuivre son effort, elle sait, par différents signaux intéroceptifs, que son objectif d'entrainement est déjà atteint. 


\subsection{Des savoir-faire perceptifs différents pour Emmi et Maeva}

Ces deux études de cas montrent des différences de perception des sensations corporelles, des différences de jugement, et enfin d'interprétation, notamment lors de l'articulation des informations internes et externes. À chaque leçon, Emmi et Maeva utilisent des échelles de ressentis identiques qui reposent sur des indicateurs tels que l'état de fatigue général, les ressentis musculaires et articulaires, la sensation de chaleur et les ressentis respiratoires. Néanmoins, toutes deux n'ont pas le même rapport à leurs sensations corporelles. Maeva est plus à-même de percevoir ses sensations corporelles : plus nombreuses, en se référant par exemple à la sensation de chaleur, à ses ressentis musculaires, à sa respiration ou encore à son activité cardiaque ; mais aussi plus fines en mentionnant les battements de son corps qui viennent de l'intérieur du corps ou la perte de contrôle de ses jambes. Par ailleurs, elle hiérarchise et combine ses sensations pour juger de l'intensité de son effort : la perception d'une difficulté respiratoire combinée à une lourdeur des jambes est interprétée comme une intensité d'effort importante qu'il faut maintenir pour atteindre la fréquence cardiaque cible. Emmi, pour sa part, fait essentiellement référence à sa sensation globale de mouvement et à une sensation assez diffuse de chaleur qu'elle n'est pas en mesure de la décrire, ni de la localiser corporellement. Ces sensations ne lui permettent pas de juger avec fiabilité de son niveau d'engagement physique, et bien qu'essayant de se référer à des sensations antérieures, elle exprime des doutes quant à l'intensité de son effort.

Pour Emmi, certaines sensations corporelles ne sont pas encore perçues, ni hiérarchisées dans un répertoire sensible. Elle n'est pas encore tout à fait capable de se rendre attentive et d'écouter les informations sensorielles fournies par son corps, dans l'objectif de réguler son engagement physique et d'ajuster son projet d'entrainement. Pour Maeva, les sensations corporelles perçues servent déjà de point de jugement en ayant été ressenties dans d'autres circonstances (Paintendre, 2017). Elle opère une démarche d'état des lieux, à partir de repères mnésiques et sensoriels qui servent de points de comparaison (notamment construits et mobilisés en course à pied ou lors du test step). La capacité à réactualiser sans cesse les connaissances de son corps par l'exploration sensorielle (Gaillard, 2011) l'amène à construire des savoir-faire perceptifs.

La mise en relations des données mesurées et internes (exprimées par les ressentis) ne s'effectue également pas de la même manière pour les deux élèves. Le rapport à l'outil technologique diverge : élément encore nécessaire pour Emmi pour situer l'intensité de son 


\section{eJRIEPS spécial 3 (janvier 2020)}

effort, il est secondaire chez Maeva qui peut s'appuyer sur ses sensations qui l'aident à percevoir relativement précisément son niveau d'engagement physique.

\section{Discussion}

Les résultats de notre étude permettent de mettre en évidence deux éléments majeurs lors d'un apprentissage sensoriel chez le pratiquant débutant en step:

1/ une acuité perceptive variable liée à la capacité du pratiquant de percevoir finement, de hiérarchiser et de mettre en lien ses sensations corporelles au cours de la pratique ; 2/ une interprétation et un raisonnement liés à la perception des sensations corporelles reposant sur des lectures technologiques et incarnées différentes.

\subsection{Hétérogénéité sensible des pratiquants}

Nos résultats montrent une diversité des sensibilités parmi les pratiquants. Des univers sensoriels vécus et utilisés différemment sont présentés autour des cas d'Emmi et de Maeva. Les processus d'apprentissage sensoriels, révélés dans une communauté de pratique singulière (la classe) sont multiples et montrent que chacun, par un cheminement singulier, structure et organise son propre système de repérage sensoriel (cette interprétation se confirme par les dix autres élèves avec lesquels nous avons travaillé pour cette étude, voir Paintendre, 2017). Cette relation singulière à son corps, construite et entretenue par le sujet, semble impacter l'organisation et l'évolution de son échelle de sensibilité. Les sensations corporelles deviennent progressivement des outils plus ou moins efficaces et pertinents à la régulation du projet de pratique en step. Nos résultats révèlent combien cette sensibilité en tant que disposition de l'homme à être affecté par ses sens, peut se modeler et s'ajuster sans cesse face à différents environnements (Récopé, 2001). En effet, l'environnement dans lequel évolue Emmi et Maeva lors de leur activité est sans doute imprégné par la nature de leur projet d'entrainement (puissance ou capacité aérobie) mais aussi par les outils mis à disposition par l'enseignante (échelle de ressentis, cardiofréquencemètre) et favorise des jugements, un recours à des connaissances antérieures fondées sur des sensations corporelles et l'émergence de nouvelles connaissances sensibles (Barberousse, 1999) plus ou moins fines, fiables et incorporées (Huet \& Gal-Petitfaux, 2011). La préoccupation d'écouter son corps, comme le montre Maeva en faisant fi d'une lecture de son cardiofréquencemètre, représente une forme d'exploration active de son corps en mouvement (Berthoz, 2013). En devenant progressivement un « visiteur assidu » (Da Silva, 2013, p. 59) et surtout volontaire de son 


\section{eJRIEPS spécial 3 (janvier 2020)}

corps, elle affine sa sensibilité par la perception de sensations corporelles diverses (respiratoire, musculaire, cardiaque).

\subsection{Du savoir-faire perceptif à la compétence perceptive et sensible}

Nos résultats montrent que les pratiquants apprennent à se rendre attentifs à certains signaux corporels (chaleur corporelle, tiraillements musculaires, accélération du cœur, essoufflement, etc.) ; ils apprennent, à leur rythme, à les repérer, à les identifier, à les nommer, à les discriminer, à leur donner du sens en utilisant certains outils comme le cardiofréquencemètre ou des médiateurs langagiers telle l'échelle de ressentis. Ces résultats permettent de documenter la manière dont les pratiquants construisent des savoirfaire perceptifs, entendus comme la capacité à écouter et interpréter les sensations qui émanent de leur corps, afin de mieux gérer leur effort physique et leur performance. L'adaptation du pratiquant à son environnement lors de sa pratique corporelle est ainsi déterminée par sa capacité à tendre vers une "hyper-écoute de son corps » (Schirrer, 2018) bien que celle-ci varie selon la fiabilité perçue des informations sensorielles et I'utilisation variée des informations du cardiofréquencemètre. II s'agit de constituer sa propre échelle de ressentis (Schirrer \& Paintendre, 2017; Paintendre et al., 2019) menant vers de nouvelles configurations sensorielles.

Nous pouvons alors nous interroger sur la pertinence d'une notion de compétence perceptive et sensible, susceptible d'imprégner le domaine sportif et scolaire, et d'enrichir les stratégies d'intervention. En effet, la compétence est définie, dans le monde scolaire, comme l'aptitude à accomplir une tâche ou faire face à des situations complexes ou inédites (BO n¹7 du 23 avril 2015). Le sujet compétent est capable de faire preuve de transversalité entre différentes situations et expériences vécues. La compétence articule différents types de ressources (connaissances, attitudes, capacités) et se complexifie sans cesse dans une classe de situations particulières (Delignières, 2009). Par conséquent, la compétence perceptive et sensible englobe et dépasse les savoir-faire perceptifs. Les savoir-faire perceptifs sont une composante de la compétence perceptive et sensible au même titre que tout un ensemble de capacités et ressources à la disposition du sujet. Inscrite dans la complexité d'un environnement incertain, étendue à des espaces de pratiques diversifiés, la compétence perceptive et sensible suppose la mobilisation et l'articulation d'informations nombreuses (notamment des données corporelles subjectives, des données corporelles objectives, des savoirs objectivés, des éléments de l'environnement d'évolution). En effet, pour Bessy et Chateauraynaud (1995), la compétence perceptive consisterait à « savoir 


\section{eJRIEPS spécial 3 (janvier 2020)}

articuler des épreuves sensorielles avec des raisonnements fondés sur des indices et repères dotés de significations dans un espace de représentation donné ».

Les pratiquants observés apprennent progressivement à mettre en relation des données subjectives (leurs ressentis corporels) dotées de significations, des données objectives concernant leur pratique (performance, atteinte de l'objectif visé en termes de vitesse, durée d'utilisation de paramètres énergétiques et biomécaniques), des savoirs, des données corporelles objectives mesurées (fréquence cardiaque). Cette articulation leur permet d'agir et de réguler leur activité en portant des jugements plus ou moins fins sur l'intensité de leur effort, la détente de leur corps, le caractère ordinaire bien que désagréable de certains ressentis, les signes précurseurs d'un danger, comme le montrent les travaux en apnée avec l'exemple de la syncope qui doivent amener à stopper la pratique (Schirrer, 2018). Les pratiquants établissent aussi des liens avec des perceptions sensorielles vécues au cours d'autres expériences corporelles. Parmi les élèves, certains avaient déjà construit des échelles de repères, dans d'autres activités physiques, et transposaient ces repères et raisonnements en step (e.g., Maeva et la course à pied).

Nous pouvons finalement avancer qu'une compétence perceptive et sensible consiste à savoir articuler des épreuves sensorielles avec des raisonnements fondés sur des indices, et repères. Elle s'exprime à l'épreuve du réel, dans des espaces diversifiés de pratique et renvoie à cette capacité d'écouter son corps, percevoir et donner du sens à des sensations, pour juger, décider et agir. Le développement de cette compétence perceptive et sensible constitue un enjeu d'importance pour le développement de la personne.

\section{Pistes d'intervention pour développer des compétences perceptives}

Les savoirs, certains outils technologiques, mais aussi les pairs (intervenants, autres pratiquants) peuvent être des médiateurs du développement de compétences perceptives et sensibles (Schirrer, 2018).

\subsection{Des outils pour investiguer et focaliser son attention sur les sensations corporelles}

L'élève/pratiquant développe ses compétences perceptives et sensibles par une écoute attentive et séquentielle de ses manifestations corporelles. L'intervenant peut inviter le pratiquant à trouver les étapes ou focalisations successives les plus pertinentes par rapport à son projet d'action (zones de corps ou domaines de sensations : respiratoires, musculaires, tonicité) (Schirrer, 2015). En effet, la perception des sensations corporelles 


\section{eJRIEPS spécial 3 (janvier 2020)}

revêt une complexité plus ou moins forte pour le pratiquant qu'il convient de guider vers une meilleure compréhension et analyse de ses ressentis (avec l'intervenant, le groupe de pairs, à l'aide de connaissances ou encore d'outils) (Paintendre, 2017).

En EPS, l'échelle de ressentis représente le premier outil constitué pour que l'attention de l'élève prenne la direction d'une écoute de soi et établisse une première "relation à soi " (Bois \& Austry, 2007). Elle permet de dépasser l'analyse binaire de son ressenti. Elle offre l'opportunité à l'élève de se focaliser sur soi (après chaque répétition en step par exemple). Cet outil construit par l'enseignant peut s'affiner peu à peu en invitant l'élève à sa coconstruction, avec ses propres mots et repères par exemple. II s'agit alors d'un accompagnement à la conscientisation des effets de la pratique sur soi où l'enjeu serait d'abord de s'ouvrir davantage à soi et certaines sensations non perçues pour ensuite favoriser leur interprétation fiable. En effet, cette échelle place une focale sur des thèmes comme la respiration, la chaleur, l'état musculaire et articulaire, l'état général de fatigue, en proposant une graduation ou progressivité. Sur la base d'une échelle de Borg (1970), d'émoticônes, d'une échelle descriptive (relâchement, contraction, tétanisé) ou par un couplage description qualitative / mesure chiffrée, l'enjeu de ces échelles, qui pourront être conservées dans un cahier de sensations ou d'entraînement, est d'amener chaque pratiquant à porter son attention sur l'expression singulière de la sensorialité, à travers son mouvement.

\subsection{Partager et mettre en mots ses ressentis}

Le pratiquant ne peut se contenter d'apprendre à identifier une sensation. II lui faut la relier et l'articuler à d'autres informations, pour lui donner du sens et la rendre crédible. L'intervenant peut inciter et aider à cette mise en relation progressive puis systématique, entre des ressentis, des effets ou une performance et des données objectives concernant ces effets (fréquence cardiaque). Des échanges entre pairs ou avec l'intervenant, la médiation langagière (par des métaphores ou des échelles de ressentis notamment), aident les pratiquants à analyser ce qui se passe dans leur corps (Schirrer, 2018), à accepter voire lâcher prise face à des sensations désagréables, à apprécier un niveau d'engagement physique. L'altérité aide à la constitution d'une culture sensorielle partagée sans asservir mais davantage nourrir l'acuité perceptive de chacun.

Le débat qui prend forme en anthropologie sensorielle sur la place du langage dans la perception sensorielle peut nous éclairer sur des stratégies d'intervention possibles en EPS. La dénomination vient-elle s'associer après-coup au contenu de la sensation ? Le langage 


\section{eJRIEPS spécial 3 (janvier 2020)}

permet-il la transcription plus ou moins précise des sensations ? Y a-t-il une indépendance entre la sensation et le langage ? En effet, les mots sont parfois difficiles à trouver pour exprimer des perceptions et l'anthropologie sensorielle souligne une indépendance relative de la sensation sur le langage. Selon Gélard, " une personne sait reconnaître une odeur, un goût, etc. et lui attribuer une valeur affective, sans pour autant savoir les nommer. " (2013, p. 15). Les métaphores ou des échelles de ressentis sont alors très utiles pour " dire" ces sensations corporelles inédites puisque la dénomination participe également en partie de la perception. Les mots organisent les catégories qui nous servent à connaître le monde. "La dénomination ne vient pas toujours s'associer " après coup et comme par accident » au contenu de la sensation. Elle contribuerait dans ce cas à « informer l'entrée sensorielle. » (Candau, 2005).

Aussi des temps d'échanges et d'interprétation sur les sensations ressenties, au sein de groupement affinitaires d'élèves, peuvent être mis en place, pour apprendre à reconnaître et dire ses sensations. Le partage d'expérience entre pairs aide les pratiquants à mettre des mots sur les sensations pour atteindre le mot juste et adapté à la sensation perçue. En passant par le discours, par l'écrit, par l'échange, par le dessin, le partage des ressentis peut être une voie d'accès à la perception et l'affinement des sensations, et à leurs interprétations (Terré, 2015). L'apport de connaissances physiologiques ou biomécaniques peuvent également guider le pratiquant dans cette mise en mots, et dans la compréhension de ses ressentis en comparaison aux données objectives. Ces connaissances permettent d'insérer les (nouvelles) sensations dans un réseau de significations.

Ainsi, partager les ressentis, chercher à les décrypter, y mettre des mots et du sens apparaît nécessaire. Ce travail collaboratif refonde une démarche d'enseignement s'appuyant sur des communautés sensorielles, facilitant la co-construction d'une "langue du corps » (Andrieu, 2014b). Si les perceptions sont orientées par l'acuité perceptive singulière de l'individu, la culture sensorielle co-construite et partagée par l'intermédiaire du langage favorise également leur accès et interprétation.

\subsection{Confronter lecture incarnée et technologique}

Les technologies numériques constituent des moyens pour mesurer l'activité et apporter des feedbacks au pratiquant. À priori fiables, objectifs et rigoureux, des outils de mesure technologique tel que le cardiofréquencemètre, enrichissent les pouvoirs de lecture du pratiquant par une préhension objectivée des données corporelles. Néanmoins, les premières informations émanent du corps en action avant même que le sujet n'ait pris 


\section{eJRIEPS spécial 3 (janvier 2020)}

l'information visuelle sur l'instrument technologique. L'acte de percevoir, conjugué à l'acte de relever des données chiffrées serait un chemin pour atteindre une première expertise perceptive (Paintendre \& Andrieu, 2015). Cependant l'écoute et la lecture de son corps ne vont pas de soi pour l'adolescent, et sont parfois même dévalorisées. Dans la réalité des cours d'EPS, une hétérogénéité sensible, qui se traduit par des différences de perception, jugement et interprétation des sensations corporelles, questionne l'utilisation des feedbacks extéroceptif et intéroceptif. La mesure chiffrée supplante-t-elle désormais les informations internes du corps (Verchère, 2016) ? Ou au contraire, les « impressions » ou ressentis valorisent-ils la sensibilité comme capacité de jugement pour ajuster son engagement dans la pratique ? Comment aider élèves et pratiquants dans cette mise en relation de données objectives et subjectives, dans cette mise en synergie d'une lecture incarnée et technologique de son corps en mouvement pour favoriser au développement d'une compétence perceptive et sensible?

Si Maeva est capable de percevoir et de se fier à des informations sensorielles internes en dépassant l'utilisation du cardiofréquencemètre en fin de séquence d'apprentissage, c'est sans doute parce qu'elle a opéré une comparaison systématique entre une fréquence cardiaque objectivée et des sensations corporelles perçues. Son échelle de sensibilité s'affine par la mise en tension de ces deux types de données. L'outil de mesures renseigne le pratiquant sur son activité et peut alors devenir support à des liens entre la perception de son effort et l'effort réellement produit. À l'inverse, les ressentis devraient pouvoir être discutés voire contrariés pour éviter des écarts importants entre ce qui est perçu par le pratiquant et l'intensité physique réelle (e.g., Emmi). Les données objectivées complètent, parfois confirment des ressentis, ou encore sont prétextes à l'exploration de ressentis associés. Par une tension entre deux formes de lectures du corps, incarnée et technologique, les jugements perceptifs se fondent et contribuent à l'affinement de repères sur soi et en soi en lien avec une technique recherchée, pour positionner son corps dans l'espace, pour réguler son intensité d'effort.

\subsection{Amplifier, extrémiser, contraster : une ébullition sensorielle}

La posture active de «présence attentive » à ses sensations, ou « awareness » (Fourure, 2004), est plus ou moins aisée selon les acteurs. L'utilisation de contrastes, sources de sensations différentes, voire « extrémisées », pourrait faciliter leur perception (Schirrer, 2015) : par exemple vivre un effort physique intense (travail en puissance aérobie, travail en anaérobie lactique par exemple), ou agir dans des conditions aménagées suscitant 


\section{eJRIEPS spécial 3 (janvier 2020)}

l'ébullition des sens (e.g., enchaîner des apnées dynamiques de $25 \mathrm{~m}$, avec très peu de récupération entre chaque apnée, de quelques secondes à un seul cycle ventilatoire, amplifie l'impression de soif d'air; enchaîner deux voies en moulinette afin de rendre indispensable l'utilisation des jambes et soulager le travail des bras).

Par ces situations contrastées jouant sur des paramètres de l'activité, le pratiquant perçoit plus aisément un certain nombre de sensations. Ainsi repérées, elles sont analysées (avec l'aide de l'intervenant ou de ses pairs). Mises en relation avec ces paramètres, elles lui permettent d'affiner ses perceptions, de réguler son action ou son effort. Ces situations contrastées contribuent à la construction d'une compétence perceptive et sensible, en faisant vivre au pratiquant des moments forts et mémorables (une intensité d'effort, la détente du corps, le caractère désagréable de certains ressentis, les signes précurseurs d'un danger). Ces marqueurs incorporés dans une situation extrêmisée permettent au pratiquant d'établir des liens avec des perceptions sensorielles vécues au cours d'autres expériences corporelles, telle que l'exprime Maeva en comparant des sensations perçues en course à pied et en step. Ainsi, à l'épreuve d'un réel amplifié, dans des espaces diversifiés de pratique, l'élève/pratiquant apprend à écouter son corps, percevoir et donner du sens à des sensations, pour juger, décider et agir.

\section{Conclusion}

Les apprentissages sensoriels apparaissent ici comme une "plus-value » d'une éducation par le sport ou l'Éducation Physique, renforçant l'autonomie des acteurs dans la régulation de leur propre activité physique ou de leurs performances sportives. En effet, dans une " société moderne subjectiviste » (Bajoit, 2013) à l'hyper-responsabilisation des sujets, les offres connectées d'assistance et d'accompagnement de la lecture de son corps envahissent les espaces de pratique physique. Néanmoins, il s'agit d'aider les pratiquants à devenir " agents de soi " (Andrieu, 2012), d'accompagner l'attention aux données sensorielles qui accompagnent toute expérience corporelle, afin de mieux respecter ou développer les possibilités de son corps. Vivre ici et maintenant, se connecter au présent et à son expérience corporelle, semblent des enjeux majeurs qui ne doivent pas être confondus avec un repli sur soi. Le partage et la co-construction nous semblent alors essentiels, alors que l'usage des outils technologiques de lecture du corps doit être interrogé. 


\section{Bibliographie}

Andrieu, B. (2017). Apprendre de son corps. Une méthode émersive au CNAC. Rouen : Presses Universitaires.

Andrieu, B. (2014). Donner le vertige. Les arts immersifs. Montréal : Editions Liber.

Andrieu, B. (2014b). Le corps-chronique en première personne : s'autosanter par les récits de sa maladie. Thérapie psychomotrice et recherches, 77 (47), 4-15.

Andrieu, B. (2012). L'Autosanté. Vers une médecine réflexive. Paris : Armand Colin.

Bajoit, G. (2013). L'individu sujet de lui-même. Vers une socio-analyse de la relation sociale.

Paris : Armand Colin.

Barberousse, A. (1999). L'expérience. Paris : Edition Flammarion.

Becker, H. (2012, 1 ${ }^{\text {ère }}$ éd. 1963). Outsiders. Etudes de sociologie de la déviance. Paris : Métailié.

Berthoz, A. (2013, 1 ère éd. 1997). Le sens du mouvement. Paris : Odile Jacob.

Bessy, C., \& Chateauraynaud, F. (1995). Experts et faussaires. Pour une sociologie de la perception. Paris : Métailié.

Bois, D., \& Austry, D. (2007). Vers l'émergence du paradigme du sensible. Réciprocités. Introduction au paradigme du sensible, [en ligne]. Page consultée de http://www.cerap.org/sites/default/files/publicdownloads/articles reciprocites/DB D

A paradigme sensible.pdf

Borg, G. (1970). Perceveid Exertion as an indicator of somatic stress. Scand J Rehabil Med, 2, 92-98.

Boulnois, I., \& Kogut, P. (2014). Step et connaissance de soi. Revue EP\&S, 361, 66-70.

Bouveresse, J. (1995). Langage, perception et réalité. Nîmes : Jacqueline Chambon.

Candau, J. (2005). Vin, arômes, couleurs et descripteurs sensoriels. Quel partage de la dégustation? Médiation \& Information, 23, 23-38.

Collinet, C., Terral, P., \& Trabal, P. (2016). Les formes de travail scientifique en STAPS : entre disciplinarité et interdisciplinarité. Movement \& Sport Sciences, 94, 41-53.

Coquart, J-B-J., Lensel, G., \& Garcin, M. (2009). Perception de l'effort chez l'enfant et l'adolescent : mesure et intérêts. Science \& Sports, 24, 137-145.

Craig, A-D. (2003). Interoception: the sense of the physiological condition of the body. Current Opinion in Neurobiology, 13, 500-505.

Craig, A-D. (2004). Human feelings: why are some more aware than others? Cognitive Sciences, 8(6), 239-241. 


\section{eJRIEPS spécial 3 (janvier 2020)}

Da Silva, M-C-M. (2013). La psychopédagogie perceptive et son influence sur ma transformation professionnelle en tant qu'enseignante, Réciprocités, 8, CERAP.

Delignières, D. (2009). Complexité et compétences. Paris : Editions EP\&S.

Farb, N-A., Segal, Z-V., \& Anderson, A-K. (2012). Mindfulness meditation training alters cortical representations of interoceptive attention. Soc. Cogn. Affect.Neurosci., 8, $15-26$.

Fourure, S. (2004). Awareness and consciousness. Gestalt, 27(2), 12.

Gaillard, J. (2011). Apprentissage technique et prise en compte de la sensibilité. In B. Huet, et N. Gal-Petitfaux (Eds.) L'expérience corporelle (pp. 77-96). Paris : Edition EP\&S.

Garcin, M. (2002). Effort et EPS : course de durée de la théorie à la pratique. Revue EP\&S, 297, 55-58.

Gaser, C., \& Schlaug, G. (2003). Brain structures differ between musicians and nonmusicians. J. Neurosci., 23, 9240-9245.

Gélard, M-L. (2013). Corps sensibles. Usages et langages des sens. Nancy : Presses Universitaires.

Huet, B., \& Gal-Petiffaux, N. (2011). L'inscription corporelle de l'expérience en EPS. In B. Huet, \& N. Gal-Petitfaux (Eds.), L'expérience corporelle (pp. 61-76). Paris : Edition EP\&S.

Merleau-Ponty, M. (1945). Phénoménologie de la perception. Paris : Gallimard.

MEN (2010). Enseignements commun, d'exploration et facultatif. Programme d'enseignement d'éducation physique et sportive pour les lycées d'enseignement général et technologique [en ligne], paru au BO, spécial n 4 du 29 avril. Consulté de https://www.education.gouv.fr/pid23972/special-n-4-du-29-avril-2010.html

MEN (2015). Socle commun de connaissances, de compétences et de culture [en ligne], paru au BO, $\mathrm{n}^{\circ} 17$ du 23 avril 2015. Consulté de https://www.education.gouv.fr/pid285/bulletin officiel.html?cid bo $=87834$

MEN (2018). Éducation physique et sportive. Nouveau référentiel des épreuves d'EPS organisées en contrôle en cours de formation : modification [en ligne], paru au BO, n'25 du $21 \quad$ juin. Consulté de https://www.education.gouv.fr/pid285/bulletin officiel.html?cid bo $=131576$

MEN (2019). Programme d'enseignement commun et d'enseignement optionnel d'éducation physique et sportive pour la classe de seconde générale et technologique et pour les classes de première et terminale des voies générale et 


\section{eJRIEPS spécial 3 (janvier 2020)}

technologique [en ligne] ,paru au BO spécial $n^{\circ} 1$ du 22 janvier 2019. Consulté de https://www.education.gouv.fr/pid285/le-bulletin-officiel.html?pid bo=38502

Paintendre, A., Schirrer, M., \& Andrieu, B. (2019). Développer des savoir-faire perceptifs en Education Physique et Sportive: analyse de l'activité d'élèves engagés dans une séquence d'enseignement en step. Activités, [En ligne] (16)1.

Paintendre, A. (2017). Le corps capacitaire en step: la construction de savoir-faire perceptifs au cours de l'expérience corporelle. Thèse de Doctorat de Sciences et Techniques des Activités Physiques et Artistiques. Université Paris-Descartes, Paris, France.

Paintendre, A. (2015). Step. In B. Andrieu (Ed.), Vocabulaire International de philosophie du sport, Tome 2 : les nouvelles recherches, (pp. 165-170). Paris : L'Harmattan.

Paintendre, A., \& Andrieu, B. (2015). Le corps capacitaire des adolescent(e)s: Une émersion du vivant dans leur perception du step. STAPS, 108, 49-59.

Pollatos, O., Schandry, R., Auer, D.P., \& Kaufmann, C. (2007). Brain structures mediating cardiovascular arousal and interoceptive awareness. BrainRes, 1141, 178-187.

Récopé, M. (2001). L'apprentissage. Paris : Edition EP\&S.

Sayeux, A. S. (2008). Surfeurs, l'être au monde. Une analyse socio-anthropologique. Rennes: Presses Universitaires de Rennes.

Schirrer, M. (2015). Immersion en apnée : cultures motrices et symbolismes aquatiques. Introduction. In M. Schirrer (Ed.), S'immerger en apnée : cultures motrices et symbolismes aquatiques (pp. 19-48). Paris : L'Harmattan.

Schirrer, M. (2018). Construire des savoir-faire perceptifs en apnée-Une méthode de réflexivité en action coach-pratiquant. Movement \& Sport Sciences-Science \& Motricité, 99, 35-46.

Schirrer, M., \& Paintendre, A., (2017). Rapport au corps et savoir-faire perceptif dans les activités physiques et Sportives. In G. Cogérino (Ed.) Rapport au corps, genre et réussite en EPS, (pp. 275-290). Montpellier : AFRAPS.

Terré, N. (2015). La connaissance des élèves en EP. Étude de la dynamique de construction et d'actualisation des connaissances à partir de l'expérience de lycéens à l'échelle d'un cycle d'apprentissage en escalade. Thèse de doctorat en Sciences et Techniques des Activités Physiques et Sportives. Université de Nantes, Nantes, Paris.

Theureau, J. (2006). Le cours d'action : méthode développée. Toulouse : Octarès. 


\section{eJRIEPS spécial 3 (janvier 2020)}

Theureau, J. (2010). Les entretiens d'autoconfrontation et de remise en situation par les traces matérielles et le programme de recherche " cours d'action ". Revue d'anthropologie des connaissances, 2(4), 287-322.

Vanpoulle, Y. (2013). Expérience vécue, intelligence motrice, phénoménologie et renouveaux paradigmatiques. Movement \& Sport Sciences, 81, 57-66.

Varela, F. J. (1989). Autonomie et connaissance, Paris : Le seuil.

Verchère, R. (2016). Les innovations technologiques dans le sport enrichissent-elles l'expérience corporelle ? Corps, 14, 115-122.

Wacquant, L. (1989). Corps et âme [Notes ethnographiques d'un apprenti-boxeur]. Actes de la recherche en sciences sociales, 80(1), 33-67.

Wathelet, O. (2013). Ethnographie cognitive des sens. In M.-L. Gélard (Ed.). Corps sensibles, (pp. 303-317). Nancy : PUN. 